\title{
Two Axiomatizations of Nelson Algebras
}

\author{
Adam Grabowski \\ Institute of Informatics \\ University of Białystok \\ Ciołkowskiego 1M, 15-245 Białystok \\ Poland
}

Summary. Nelson algebras were first studied by Rasiowa and BiałynickiBirula [1] under the name N-lattices or quasi-pseudo-Boolean algebras. Later, in investigations by Monteiro and Brignole [3, 4, and 2] the name "Nelson algebras" was adopted - which is now commonly used to show the correspondence with Nelson's paper [14 on constructive logic with strong negation.

By a Nelson algebra we mean an abstract algebra

$$
\langle L, \top,-, \neg, \rightarrow, \Rightarrow, \sqcup, \sqcap\rangle
$$

where $L$ is the carrier, - is a quasi-complementation (Rasiowa used the sign $\sim$, but in Mizar "-" should be used to follow the approach described in [12] and [10]), $\neg$ is a weak pseudo-complementation, $\rightarrow$ is weak relative pseudocomplementation and $\Rightarrow$ is implicative operation. $\sqcup$ and $\sqcap$ are ordinary lattice binary operations of supremum and infimum.

In this article we give the definition and basic properties of these algebras according to [16] and [15]. We start with preliminary section on quasi-Boolean algebras (i.e. de Morgan bounded lattices). Later we give the axioms in the form of Mizar adjectives with names corresponding with those in 15. As our main result we give two axiomatizations (non-equational and equational) and the full formal proof of their equivalence.

The second set of equations is rather long but it shows the logical essence of Nelson lattices. This formalization aims at the construction of algebraic model of rough sets 9 in our future submissions. Section 4 contains all items from Th. 1.2 and 1.3 (and the itemization is given in the text). In the fifth section we provide full formal proof of Th. 2.1 p. 75 [16].

MSC: 06D30 08A05 03B35

Keywords: quasi-pseudo-Boolean algebras; Nelson lattices; de Morgan lattices MML identifier: NELSON_1, version: 8.1.04 5.32.1237 
The notation and terminology used in this paper have been introduced in the following articles: [5], [6], [7], [18, [1], [13], 17], and [8].

\section{De Morgan and Quasi-Boolean Lattices}

Let $L$ be a non empty ortholattice structure. We say that $L$ is de Morgan if and only if

(Def. 1) for every elements $x, y$ of $L,(x \sqcap y)^{\mathrm{c}}=x^{\mathrm{c}} \sqcup y^{\mathrm{c}}$.

One can verify that every non empty ortholattice structure which is de Morgan and involutive is also de Morgan and every non empty ortholattice structure which is de Morgan and involutive is also de Morgan.

Every non empty ortholattice structure which is trivial is also de Morgan and there exists a non empty ortholattice structure which is de Morgan, involutive, bounded, distributive, and lattice-like.

A de Morgan algebra is a de Morgan, involutive, distributive, lattice-like, non empty ortholattice structure.

A quasi-Boolean algebra is a bounded de Morgan algebra. From now on $L$ denotes a quasi-Boolean algebra and $x, y, z$ denote elements of $L$.

Now we state the propositions:

(1) $(x \sqcup y)^{\mathrm{c}}=x^{\mathrm{c}} \sqcap y^{\mathrm{c}}$.

(2) $\left(\top_{L}\right)^{\mathrm{c}}=\perp_{L}$.

(3) $\left(\perp_{L}\right)^{\mathrm{c}}=\top_{L}$.

(4) $x \sqcap(x \sqcap y)=x \sqcap y$.

(5) $x \sqcup(x \sqcup y)=x \sqcup y$.

\section{The Structure and Operators in Nelson Algebras}

We consider Nelson structures which extend ortholattice structures and are systems

〈a carrier, a unity, a complement operation, a weak pseudo-complementation,

a weak relative pseudo-complementation, an implicative operation,

$$
\text { a join operation, a meet operation〉 }
$$

where the carrier is a set, the unity is an element of the carrier, the complement operation and the weak pseudo-complementation are unary operations on the carrier, the weak relative pseudo-complementation and the implicative operation 
and the join operation and the meet operation are binary operations on the carrier.

Note that there exists a Nelson structure which is strict and non empty and there exists a non empty Nelson structure which is trivial, de Morgan, involutive, bounded, distributive, and lattice-like.

Let $L$ be a non empty Nelson structure and $a, b$ be elements of $L$. The functor $a \rightarrow b$ yielding an element of $L$ is defined by the term

(Def. 2) (the weak relative pseudo-complementation of $L)(a, b)$.

We say that $a<b$ if and only if

(Def. 3) $a \rightarrow b=\top_{L}$.

We say that $a \leqslant b$ if and only if

(Def. 4) $a=a \sqcap b$.

Let $a$ be an element of $L$. The functor $\neg a$ yielding an element of $L$ is defined by the term

(Def. 5) (the weak pseudo-complementation of $L)(a)$.

Let $a, b$ be elements of $L$. The functor $a \Rightarrow b$ yielding an element of $L$ is defined by the term

(Def. 6) (the implicative operation of $L)(a, b)$.

\section{The Non-Equational Axiomatization}

Let $L$ be a non empty Nelson structure. We say that $L$ has reflexive $<$ if and only if

(Def. 7) for every element $a$ of $L, a<a$.

We say that $L$ has transitive $<$ if and only if

(Def. 8) for every elements $a, b, c$ of $L$ such that $a<b<c$ holds $a<c$.

Let $L$ be a bounded, lattice-like, non empty Nelson structure. We say that $L$ is quasi-Boolean if and only if

(Def. 9) $L$ is de Morgan, involutive, and distributive.

Let us note that every bounded, lattice-like, non empty Nelson structure which is quasi-Boolean is also de Morgan, involutive, and distributive.

Every bounded, lattice-like, non empty Nelson structure which is de Morgan, involutive, and distributive is also quasi-Boolean.

Let $L$ be a non empty Nelson structure. We say that $L$ satisfies $\left(\mathrm{qpB}_{3}\right)$ if and only if

(Def. 10) for every elements $x, a, b$ of $L, a \sqcap x<b$ iff $x<a \rightarrow b$.

We say that $L$ satisfies $\left(\mathrm{qpB}_{4}\right)$ if and only if 
(Def. 11) for every elements $a, b$ of $L, a \Rightarrow b=(a \rightarrow b) \sqcap(-b \rightarrow-a)$.

We say that $L$ satisfies $\left(\mathrm{qpB}_{5}\right)$ if and only if

(Def. 12) for every elements $a, b$ of $L, a \Rightarrow b=\top_{L}$ iff $a \sqcap b=a$.

We say that $L$ satisfies $\left(\mathrm{qpB}_{6}\right)$ if and only if

(Def. 13) for every elements $a, b, c$ of $L$ such that $a<c$ and $b<c$ holds $a \sqcup b<c$.

We say that $L$ satisfies $\left(\mathrm{qpB}_{7}\right)$ if and only if

(Def. 14) for every elements $a, b, c$ of $L$ such that $a<b$ and $a<c$ holds $a<b \sqcap c$.

We say that $L$ satisfies $\left(\mathrm{qpB}_{8}\right)$ if and only if

(Def. 15) for every elements $a, b$ of $L, a \sqcap-b<-(a \rightarrow b)$.

We say that $L$ satisfies $\left(\mathrm{qpB}_{9}\right)$ if and only if

(Def. 16) for every elements $a, b$ of $L,-(a \rightarrow b)<a \sqcap-b$.

We say that $L$ satisfies $\left(\mathrm{qpB}_{10}\right)$ if and only if

(Def. 17) for every element $a$ of $L, a<-\neg a$.

We say that $L$ satisfies $\left(\mathrm{qpB}_{11}\right)$ if and only if

(Def. 18) for every element $a$ of $L,-\neg a<a$.

We say that $L$ satisfies $\left(\mathrm{qpB}_{12}\right)$ if and only if

(Def. 19) for every elements $a, b$ of $L, a \sqcap-a<b$.

We say that $L$ satisfies $\left(\mathrm{qpB}_{13}\right)$ if and only if

(Def. 20) for every element $a$ of $L, \neg a=a \rightarrow-\top_{L}$.

Let us observe that there exists a bounded, lattice-like, non empty Nelson structure which is quasi-Boolean and has reflexive $<$ and transitive $<$ and satisfies $\left(q p B_{3}\right),\left(q p B_{4}\right),\left(q p B_{5}\right),\left(q p B_{6}\right),\left(q p B_{7}\right),\left(q p B_{8}\right),\left(q p B_{9}\right),\left(q p B_{10}\right),\left(q p B_{11}\right)$, $\left(q p B_{12}\right)$, and $\left(q^{p B_{13}}\right)$.

A Nelson algebra is a quasi-Boolean, bounded, lattice-like, non empty Nelson structure with reflexive $<$ and transitive $<$. Let $L$ be a bounded, non empty Nelson structure and $a, b$ be elements of $L$. Let us observe that the functor $a \Rightarrow b$ is defined by the term

(Def. 21) $(a \rightarrow b) \sqcap(-b \rightarrow-a)$.

From now on $L$ denotes a Nelson algebra and $a, b, c, d, x, y, z$ denote elements of $L$.

Now we state the propositions:

(6) $a \sqsubseteq b$ if and only if $a \leqslant b$.

(7) $a \leqslant b \leqslant a$ if and only if $a=b$.

Proof: If $a \leqslant b \leqslant a$, then $a=b$ by [18, (4), (8)].

(8) $a \sqcap b=\top_{L}$ if and only if $a=\top_{L}$ and $b=\top_{L}$. 
(9) $a \leqslant b$ if and only if $a<b$ and $-b<-a$. The theorem is a consequence of $(8)$.

(10) $a \sqcap b<a$. The theorem is a consequence of (9).

(11) $a<a \sqcup b$. The theorem is a consequence of (9).

(12) $a \leqslant b$ if and only if $a \Rightarrow b=\top_{L}$.

(13) $-(a \sqcap b)=-a \sqcup-b$. The theorem is a consequence of (1).

(14) $(a \sqcap-a) \sqcap(b \sqcup-b)=a \sqcap-a$. The theorem is a consequence of (1), (13), and (9).

(15) If $a \leqslant b \leqslant c$, then $a \leqslant c$.

(16) If $b \leqslant c$, then $a \sqcup b \leqslant a \sqcup c$ and $a \sqcap b \leqslant a \sqcap c$. The theorem is a consequence of (9), (1), and (13).

(17) $-a \sqcup b \leqslant a \rightarrow b$. The theorem is a consequence of (1), (2), (9), (10), (16), and (15).

(18) $(a \rightarrow b) \sqcap(-a \sqcup b)=-a \sqcup b$. The theorem is a consequence of (1), (13), (17), (10), (9), and (7).

(19) $-a \sqcup b<a \rightarrow b$. The theorem is a consequence of (18) and (9).

(20) $a \sqcap(a \rightarrow b)=a \sqcap(-a \sqcup b)$. The theorem is a consequence of (11), (10), (13), (1), (19), (9), and (7).

(21) If $-x<-y$, then $-(z \rightarrow x)<-(z \rightarrow y)$.

Let us assume that $x<y$. Now we state the propositions:

(22) $a \sqcap(a \rightarrow x)<y$. The theorem is a consequence of (20) and (10).

(23) $a \rightarrow x<a \rightarrow y$. The theorem is a consequence of (22).

(24) $\quad a \rightarrow(b \sqcap c)=(a \rightarrow b) \sqcap(a \rightarrow c)$. The theorem is a consequence of (11), (13), (10), (23), (9), and (7).

\section{Properties of Nelson Algebras}

Now we state the propositions:

(25) [SEe Also [16] P. 69, Th. 1.2 (5)]:

$a \Rightarrow a=\top_{L}$.

(26) [SEE ALSO [16] P. 69, TH. $1.2(6)]$ :

If $a \Rightarrow b=\top_{L}$ and $b \Rightarrow c=\top_{L}$, then $a \Rightarrow c=\top_{L}$.

(27) [SEE Also [16] P. 69, Th. $1.2(7)]$ :

If $a \Rightarrow b=\top_{L}$ and $b \Rightarrow a=\top_{L}$, then $a=b$.

(28) [SEe ALSO [16] P. 69, TH. 1.2 (8)]:

$a \Rightarrow \top_{L}=\top_{L}$. 
(29) [SEe Also [16] P. 69, Th. 1.3 (9)]:

$a \rightarrow a=\top_{L}$.

(30) [SEe Also [16] P. 69, Th. 1.3 (10)]:

If $a \rightarrow b=\top_{L}$ and $b \rightarrow c=\top_{L}$, then $a \rightarrow c=\top_{L}$.

(31) [SEE ALSO [16] P. 69, TH. 1.3 (11)]:

If $b<c$, then $a \sqcup b<a \sqcup c$ and $a \sqcap b<a \sqcap c$.

(32) [SEE Also [16] P. 69, TH. 1.3 (12)]:

If $a<b$ and $c<d$, then $a \sqcup c<b \sqcup d$ and $a \sqcap c<b \sqcap d$.

(33) [SEe ALSo [16] P. 69, TH. 1.3 (13)]:

$a \sqcap(a \rightarrow b)<b$.

(34) [SEe Also [16] P. 69, Th. 1.3 (14)]:

$a \rightarrow(b \rightarrow c)=(a \sqcap b) \rightarrow c$.

(35) [SEe Also [16] P. 69, Th. 1.3 (15)]:

$a \rightarrow(b \rightarrow c)=b \rightarrow(a \rightarrow c)$.

(36) [SEe Also [16] P. 69, Th. 1.3 (16)]:

$a<(a \rightarrow b) \rightarrow b$. The theorem is a consequence of (33).

(37) [see Also [16] P. 71, Th. 1.3 (50)]:

$a<b \rightarrow(a \sqcap b)$. The theorem is a consequence of (9).

(38) [SEe ALSO [16] P. 69, Th. 1.3 (17)]:

$a \sqcap-a \leqslant b \sqcup-b$. The theorem is a consequence of (1) and (9).

(39) [SEe Also [16] P. 70, Th. 1.3 (18)]:

$a \leqslant b \Rightarrow a \sqcap b$. The theorem is a consequence of (37) and (9).

(40) [SEe Also [16] P. 70, Th. 1.3 (19)]:

$a \rightarrow \neg b=b \rightarrow \neg a$. The theorem is a consequence of (35).

(41) [SEe Also [16] P. 70, Th. 1.3 (20)]:

$a \rightarrow \top_{L}=\top_{L}$. The theorem is a consequence of (9).

(42) [SEE ALSO [16] P. 70, Th. 1.3 (21)]:

$\perp_{L} \rightarrow a=\top_{L}$. The theorem is a consequence of (9).

(43) [SEe Also [16] P. 70, Th. 1.3 (22)]:

$\top_{L} \rightarrow b=b$. The theorem is a consequence of (9), (33), and (7).

(44) [SEe ALSo [16] P. 70, Tн. 1.3 (23)]:

If $a=\top_{L}$ and $a \rightarrow b=\top_{L}$, then $b=\top_{L}$.

(45) [SEE Also [16] P. 70, Th. 1.3 (24)]:

$a \rightarrow(b \rightarrow a)=\top_{L}$. The theorem is a consequence of (9).

(46) [SEE Also [16] P. 70, Th. 1.3 (25)]:

$(a \rightarrow(b \rightarrow c)) \rightarrow((a \rightarrow b) \rightarrow(a \rightarrow c))=\top_{L}$. The theorem is a consequence of (33) and (35). 
(47) [SEE ALSO [16] P. 70, TH. 1.3 (26)]:

$a \rightarrow(a \sqcup b)=\top_{L}$. The theorem is a consequence of (11).

(48) [SEE ALSO [16] P. 70, TH. 1.3 (27)]:

$b \rightarrow(a \sqcup b)=\top_{L}$. The theorem is a consequence of (11).

(49) [SEE ALSO [16] P. 70, TH. $1.3(28)]$ :

$(a \rightarrow c) \rightarrow((b \rightarrow c) \rightarrow((a \sqcup b) \rightarrow c))=\top_{L}$. The theorem is a consequence of (33) and (10).

(50) [SEE ALSO [16] P. 70, TH. $1.3(29)]$ :

$(a \sqcap b) \rightarrow a=\top_{L}$. The theorem is a consequence of (10).

(51) [SEE ALso [16] P. 70, TH. $1.3(30)]$ :

$(a \sqcap b) \rightarrow b=\top_{L}$. The theorem is a consequence of (10).

(52) [SEE ALSO [16] P. 70, TH. $1.3(31)]$ :

$(a \rightarrow b) \rightarrow((a \rightarrow c) \rightarrow(a \rightarrow(b \sqcap c)))=\top_{L}$. The theorem is a consequence of $(33)$.

(53) [SEE ALSO [16] P. 70, TH. $1.3(32)]$ :

$(a \rightarrow \neg b) \rightarrow(b \rightarrow \neg a)=\top_{L}$. The theorem is a consequence of $(40)$ and $(29)$.

(54) [SEE ALSO [16] P. 70, TH. 1.3 (33)]:

$\neg(a \rightarrow a) \rightarrow b=\top_{L}$. The theorem is a consequence of (29), (2), (43), and $(42)$.

(55) [SEE ALSO [16] P. 70, TH. $1.3(34)]$ :

$-a \rightarrow(a \rightarrow b)=\top_{L}$.

(56) [SEE ALSO [16] P. 70, TH. 1.3 (35)]:

$(-(a \rightarrow b) \rightarrow(a \sqcap-b)) \sqcap((a \sqcap-b) \rightarrow-(a \rightarrow b))=\top_{L}$.

(57) [SEE Also [16] P. 70, TH. 1.3 (36)]:

$(-\neg a \rightarrow a) \sqcap(a \rightarrow-\neg a)=\top_{L}$.

(58) [SEE ALSO [16] P. 70, TH. 1.3 (37)]:

$-a=a$.

(59) [SEE ALSO [16] P. 70, TH. 1.3 (38)]:

$-(a \sqcup b)=-a \sqcap-b$.

(60) [SEE ALSO [16] P. 70, TH. $1.3(39)]$ :

$-(a \sqcap b)=-a \sqcup-b$. The theorem is a consequence of (1).

(61) [SEE ALSO [16] P. 70, TH. $1.3(40)]$ :

If $a<b$, then $b \rightarrow c<a \rightarrow c$ and $c \rightarrow a<c \rightarrow b$. The theorem is a consequence of (43), (46), (10), and (41).

(62) [SEE ALSO [16] P. 70, TH. $1.3(41)]$ :

$(a \rightarrow b) \rightarrow((c \rightarrow d) \rightarrow((a \sqcap c) \rightarrow(b \sqcap d)))=\top_{L}$. The theorem is a consequence of $(33)$. 
(63) [SEE ALSO [16] P. 70, TH. 1.3 (42)]:

$(a \rightarrow b) \rightarrow((c \rightarrow d) \rightarrow((a \sqcup c) \rightarrow(b \sqcup d)))=\top_{L}$. The theorem is a consequence of $(10)$.

(64) [SEE ALSO [16] P. 70, TH. 1.3 (43)]:

$(b \rightarrow a) \rightarrow((c \rightarrow d) \rightarrow((a \rightarrow c) \rightarrow(b \rightarrow d)))=\top_{L}$. The theorem is a consequence of $(33)$.

\section{Alternative Equational Axiomatics by Rasiowa}

Let $L$ be a non empty Nelson structure. We say that $L$ satisfies (qpB $\left.{ }_{0}^{*}\right)$ if and only if

(Def. 22) for every elements $a, b$ of $L, a \leqslant b$ iff $a \Rightarrow b=\top_{L}$.

We say that $L$ satisfies $\left(\mathrm{qpB}_{1}^{*}\right)$ if and only if

(Def. 23) for every elements $a, b$ of $L, a \rightarrow(b \rightarrow a)=\top_{L}$.

We say that $L$ satisfies $\left(\mathrm{qpB}_{2}^{*}\right)$ if and only if

(Def. 24) for every elements $a, b, c$ of $L,(a \rightarrow(b \rightarrow c)) \rightarrow((a \rightarrow b) \rightarrow(a \rightarrow c))=\top_{L}$.

We say that $L$ satisfies $\left(\mathrm{qpB}_{3}^{*}\right)$ if and only if

(Def. 25) for every element $a$ of $L, \top_{L} \rightarrow a=a$.

We say that $L$ satisfies $\left(\mathrm{qpB}_{5}^{*}\right)$ if and only if

(Def. 26) for every elements $a, b$ of $L,(a \Rightarrow b) \rightarrow((b \Rightarrow a) \rightarrow b)=(b \Rightarrow a) \rightarrow((a \Rightarrow$ b) $\rightarrow a)$.

We say that $L$ satisfies $\left(\mathrm{qpB}_{6}^{*}\right)$ if and only if

(Def. 27) for every elements $a, b$ of $L, a \rightarrow(a \sqcup b)=\top_{L}$.

We say that $L$ satisfies $\left(\mathrm{qpB}_{7}^{*}\right)$ if and only if

(Def. 28) for every elements $a, b$ of $L, b \rightarrow(a \sqcup b)=\top_{L}$.

We say that $L$ satisfies $\left(\mathrm{qpB}_{8}^{*}\right)$ if and only if

(Def. 29) for every elements $a, b, c$ of $L,(a \rightarrow c) \rightarrow((b \rightarrow c) \rightarrow((a \sqcup b) \rightarrow c))=\top_{L}$.

We say that $L$ satisfies $\left(\mathrm{qpB}_{9}^{*}\right)$ if and only if

(Def. 30) for every elements $a, b$ of $L,(a \sqcap b) \rightarrow a=\top_{L}$.

We say that $L$ satisfies $\left(\mathrm{qpB}_{10}^{*}\right)$ if and only if

(Def. 31) for every elements $a, b$ of $L,(a \sqcap b) \rightarrow b=\top_{L}$.

We say that $L$ satisfies $\left(\mathrm{qpB}_{11}^{*}\right)$ if and only if

(Def. 32) for every elements $a, b, c$ of $L,(a \rightarrow b) \rightarrow((a \rightarrow c) \rightarrow(a \rightarrow(b \sqcap c)))=\top_{L}$.

We say that $L$ satisfies $\left(\mathrm{qpB}_{12}^{*}\right)$ if and only if

(Def. 33) for every elements $a, b$ of $L,(a \rightarrow \neg b) \rightarrow(b \rightarrow \neg a)=\top_{L}$.

We say that $L$ satisfies $\left(\mathrm{qpB}_{13}^{*}\right)$ if and only if 
(Def. 34) for every elements $a, b$ of $L, \neg(a \rightarrow a) \rightarrow b=\top_{L}$.

We say that $L$ satisfies $\left(\mathrm{qpB}_{14}^{*}\right)$ if and only if

(Def. 35) for every elements $a, b$ of $L,-a \rightarrow(a \rightarrow b)=\top_{L}$.

We say that $L$ satisfies $\left(\mathrm{qpB}_{15}^{*}\right)$ if and only if

(Def. 36) for every elements $a, b$ of $L,(-(a \rightarrow b) \rightarrow(a \sqcap-b)) \sqcap((a \sqcap-b) \rightarrow-(a \rightarrow b))=$ $\top_{L}$.

We say that $L$ satisfies $\left(\mathrm{qpB}_{17}^{*}\right)$ if and only if

(Def. 37) for every elements $a, b$ of $L,-(a \sqcup b)=-a \sqcap-b$.

We say that $L$ satisfies $\left(\mathrm{qpB}_{19}^{*}\right)$ if and only if

(Def. 38) for every element $a$ of $L,(-\neg a \rightarrow a) \sqcap(a \rightarrow-\neg a)=\top_{L}$.

We introduce $L$ satisfies $\left(\mathrm{qpB}_{4}^{*}\right)$ as a synonym of $L$ satisfies $\left(\mathrm{qpB}_{4}\right)$ and $L$ satisfies $\left(\mathrm{qpB}_{16}^{*}\right)$ as a synonym of $L$ is de Morgan and $L$ satisfies $\left(\mathrm{qpB}_{18}^{*}\right)$ as a synonym of $L$ is involutive.

Note that every Nelson algebra satisfies $\left(q p B_{1}^{*}\right),\left(q p B_{2}^{*}\right),\left(q p B_{3}^{*}\right),\left(q p B_{4}^{*}\right)$, $\left(q p B_{5}^{*}\right),\left(q p B_{6}^{*}\right),\left(q p B_{7}^{*}\right),\left(q p B_{8}^{*}\right),\left(q p B_{9}^{*}\right),\left(q B_{10}^{*}\right),\left(q p B_{11}^{*}\right),\left(q p B_{12}^{*}\right),\left(q p B_{13}^{*}\right)$, $\left(q \mathrm{pB}_{14}^{*}\right),\left(\mathrm{qpB}_{15}^{*}\right),\left(\mathrm{qpB}_{16}^{*}\right),\left(\mathrm{qpB}_{17}^{*}\right),\left(\mathrm{qpB}_{18}^{*}\right)$, and $\left(\mathrm{qpB}_{19}^{*}\right)$.

Now we state the proposition:

(65) Let us consider a non empty Nelson structure $L$. Suppose $L$ satisfies $\left(\mathrm{qpB}_{0}^{*}\right)$. Then $L$ is a Nelson algebra if and only if $L$ satisfies (qpB $\left.\mathrm{q}_{1}^{*}\right)$, $\left(q p B_{2}^{*}\right),\left(q p B_{3}^{*}\right),\left(q p B_{4}^{*}\right),\left(q p B_{5}^{*}\right),\left(q p B_{6}^{*}\right),\left(q p B_{7}^{*}\right),\left(q p B_{8}^{*}\right),\left(q p B_{9}^{*}\right),\left(q p B_{10}^{*}\right)$, $\left(q p B_{11}^{*}\right),\left(q p B_{12}^{*}\right),\left(q p B_{13}^{*}\right),\left(q p B_{14}^{*}\right),\left(q p B_{15}^{*}\right),\left(q p B_{16}^{*}\right),\left(q p B_{17}^{*}\right),\left(q p B_{18}^{*}\right)$, and $\left(\mathrm{qpB}_{19}^{*}\right)$.

Proof: Reconsider $L_{1}=L$ as a de Morgan, non empty Nelson structure. For every elements $a, b$ of $L_{1}$ such that $a \sqcap b=\top_{L_{1}}$ holds $a=\top_{L_{1}}$ and $b=\top_{L_{1}}$. For every elements $a, b$ of $L_{1}, a \leqslant b$ iff $a<b$ and $-b<-a$. Set $d=\left(\top_{L}\right)^{\mathrm{c}}$. For every element $a$ of $L, a \leqslant \top_{L}$. For every element $a$ of $L$, $d \leqslant a$. For every element $a$ of $L, d \sqcap a=d$. For every element $a$ of $L_{1}$, $a \rightarrow \top_{L_{1}}=\top_{L_{1}}$. For every elements $a, b, c$ of $L_{1}$ such that $a \rightarrow b=\top_{L_{1}}$ and $b \rightarrow c=\top_{L_{1}}$ holds $a \rightarrow c=\top_{L_{1}} . L_{1}$ has transitive $<. L_{1}$ satisfies $\left(\mathrm{qpB}_{6}\right)$. For every element $a$ of $L_{1}, a \rightarrow a=\top_{L_{1}}$. $L_{1}$ satisfies $\left(\mathrm{qpB}_{7}\right)$. For every elements $a, b$ of $L_{1}, a \sqcap b \leqslant a$. For every elements $a, b$ of $L_{1}, a \leqslant a \sqcup b$. For every elements $a, b$ of $L_{1}, b \leqslant a \sqcup b$. For every elements $a, b$ of $L_{1}$, $a \sqcap b \leqslant b$. For every element $a$ of $L_{1}, a \Rightarrow a=\top_{L_{1}}$. For every elements $a$, $b$ of $L_{1}, a=b$ iff $a \Rightarrow b=\top_{L_{1}}$ and $b \Rightarrow a=\top_{L_{1}}$. For every elements $a$, $b$ of $L_{1}, a \leqslant b \leqslant a$ iff $a=b$. $L_{1}$ has reflexive $<$. For every elements $a, b$, $c$ of $L_{1}$ such that $a<b$ holds $b \rightarrow c<a \rightarrow c$ and $c \rightarrow a<c \rightarrow b$. For every elements $a, b$ of $L_{1}, a \rightarrow(b \rightarrow(a \sqcap b))=\top_{L_{1}}$. For every elements $a, b, c$ of $L_{1}$ such that $a<b \rightarrow c$ holds $b<a \rightarrow c$. For every elements $a, c$ of $L_{1}$, $a \rightarrow(a \rightarrow c)<a \rightarrow c . L_{1}$ satisfies $\left(\mathrm{qpB}_{3}\right)$. For every elements $a, b, c$ of $L_{1}$ 
such that $b<c$ holds $a \sqcap b<a \sqcap c$. For every elements $a, b, c$ of $L_{1}$ such that $b<c$ holds $a \sqcup b<a \sqcup c$. For every elements $a, b, c$ of $L_{1}$ such that $a \leqslant c$ and $b \leqslant c$ holds $a \sqcup b \leqslant c$. For every elements $a, b, c$ of $L_{1}$ such that $c \leqslant a$ and $c \leqslant b$ holds $c \leqslant a \sqcap b$. For every elements $a, b$ of $L_{1}, b \sqcup a \leqslant a \sqcup b$. For every elements $a, b$ of $L_{1}, a \sqcup b=b \sqcup a$. For every elements $a, b$ of $L_{1}$, $a \sqcap b \leqslant b \sqcap a$. For every elements $a, b$ of $L_{1}, a \sqcap b=b \sqcap a$. For every elements $a, b, c$ of $L_{1}$ such that $a \leqslant b$ holds $a \sqcup c \leqslant b \sqcup c$. For every elements $a, b$ of $L_{1}, b=(a \sqcap b) \sqcup b$. For every elements $a, b$ of $L_{1}, a \sqcap(a \sqcup b)=a$. For every elements $a, b, c$ of $L_{1}$ such that $b \leqslant c$ holds $a \sqcap b \leqslant a \sqcap c$. For every elements $a, b, c$ of $L_{1}$ such that $a \leqslant b \leqslant c$ holds $a \leqslant c$. For every elements $a, b, c$ of $L_{1}, a \sqcap(b \sqcap c)=(a \sqcap b) \sqcap c$. For every elements $a, b, c$ of $L_{1}$, $a \sqcup(b \sqcup c)=(a \sqcup b) \sqcup c$. Set $c=\top_{L_{1}}$. For every element $a$ of $L_{1}, c \sqcup a=c$ and $a \sqcup c=c$ by [18, (4)]. $L_{1}$ is distributive. $L_{1}$ satisfies $\left(q_{p} B_{5}\right) . L_{1}$ satisfies $\left(q \mathrm{pB}_{8}\right) . L_{1}$ satisfies $\left(\mathrm{qpB}_{9}\right) . L_{1}$ satisfies $\left(\mathrm{qpB}_{10}\right) . L_{1}$ satisfies $\left(\mathrm{qpB}_{11}\right) . L_{1}$ satisfies $\left(\mathrm{qpB}_{12}\right)$. For every elements $a, b, c$ of $L_{1}, \neg \top_{L_{1}}=-\top_{L_{1}}$. For every elements $a, b$ of $L_{1}, a \rightarrow \neg b=b \rightarrow \neg a$. $L_{1}$ satisfies $\left(\mathrm{qpB}_{13}\right)$.

\section{REFERENCES}

[1] Andrzej Białynicki-Birula and Helena Rasiowa. On the representation of quasi-Boolean algebras. Bulletin de l'Academie Polonaise des Sciences, 5:259-261, 1957.

[2] Diana Brignole. Equational characterization of Nelson algebra. Notre Dame Journal of Formal Logic, (3):285-297, 1969.

[3] Diana Brignole and Antonio Monteiro. Caracterisation des algèbres de Nelson par des egalités, I. Proceedings of the Japan Academy, 43(4):279-283, 1967. doi $10.3792 / \mathrm{pja} / 1195521624$.

[4] Diana Brignole and Antonio Monteiro. Caracterisation des algèbres de Nelson par des egalités, II. Proceedings of the Japan Academy, 43(4):284-285, 1967. doi $10.3792 / \mathrm{pja} / 1195521625$.

[5] Czesław Byliński. Binary operations. Formalized Mathematics, 1(1):175-180, 1990.

[6] Czesław Byliński. Functions and their basic properties. Formalized Mathematics, 1(1): 55-65, 1990.

[7] Czesław Byliński. Functions from a set to a set Formalized Mathematics, 1(1):153-164, 1990.

[8] Czesław Byliński. Some basic properties of sets Formalized Mathematics, 1(1):47-53, 1990.

[9] Adam Grabowski. Automated discovery of properties of rough sets. Fundamenta Informaticae, 128:65-79, 2013. doi 10.3233/FI-2013-933

[10] Adam Grabowski. Mechanizing complemented lattices within Mizar system. Journal of Automated Reasoning, 2015. doi $10.1007 / \mathrm{s} 10817-015-9333-5$.

[11] Adam Grabowski. Robbins algebras vs. Boolean algebras Formalized Mathematics, 9(4): 681-690, 2001.

[12] Adam Grabowski and Markus Moschner. Managing heterogeneous theories within a mathematical knowledge repository. In Andrea Asperti, Grzegorz Bancerek, and Andrzej Trybulec, editors, Mathematical Knowledge Management Proceedings, volume 3119 of Lecture Notes in Computer Science, pages 116-129. Springer, 2004. doi:10.1007/978-3540-27818-4_9. 3rd International Conference on Mathematical Knowledge Management, Bialowieza, Poland, Sep. 19-21, 2004.

[13] Adam Grabowski and Markus Moschner. Formalization of ortholattices via orthoposets 
Formalized Mathematics, 13(1):189-197, 2005.

[14] David Nelson. Constructible falsity. Journal of Symbolic Logic, 14:16-26, 1949.

[15] Helena Rasiowa. Algebraic Models of Logics. Warsaw University, 2001.

[16] Helena Rasiowa. An Algebraic Approach to Non-Classical Logics. North Holland, 1974.

[17] Zinaida Trybulec. Properties of subsets Formalized Mathematics, 1(1):67-71, 1990.

[18] Stanisław Żukowski. Introduction to lattice theory Formalized Mathematics, 1(1):215222, 1990.

Received April 19, 2015 